\title{
Collecte des données des médecins: rôle et responsabilité des cantons
}

\author{
Des données d'économie de la santé solides constituent la base d'une structure tari- \\ faire uniforme dans le système de santé suisse. Dans le cadre de la conférence «Col- \\ lecte des données des médecins: rôle et responsabilité des cantons» du 29.3.2012, \\ Newlndex a présenté le projet OBELISC aux responsables des sociétés cantonales de \\ médecine et des TrustCenters.
}

Renato Laffranchi, Philip Moline

NewIndex AG

Correspondance: Dr Renato Laffranchi

NewIndex AG

Leberngasse 19

CH-4600 Olten

renato.laffranchi[at]newindex.ch
NewIndex AG collecte depuis 2004 les données de facturation des médecins libres praticiens en Suisse. Ces données, fournies par chaque médecin par le biais du TrustCenter, sont agrégées (condensées) et servent de base au miroir du cabinet. Chaque médecin a ainsi la possibilité de se comparer aux collègues de sa discipline dans un système de référence, et aussi de mieux se protéger contre les réclamations injustifiées des assureurs. A l'échelle de la Suisse, 10 TrustCenters traitent les données des médecins créant ainsi un pool de données utilisé comme instrument dans les négociations tarifaires avec les assureurs et disponible en cas de procédure d'économicité, a souligné Andreas Häfeli, président du conseil d'administration de NewIndex.

Urs Stoffel, président de la Société cantonale de médecine de Zurich, met l'accent sur l'importance des données anonymisées, consolidées et agrégées que NewIndex exploite et analyse. «Ces données sont indispensables au contrôle de plausibilité et au pilotage de la Convention prestations-prix (CPP) avec les assureurs, qui à son tour est la base de la fixation de la valeur de point tarifaire.»

\footnotetext{
Newlndex AG

L'entreprise Newlndex a été fondée en 2000 dans la perspective des exigences tarifaires du TARMED et est détenue à $100 \%$ par les sociétés cantonales de médecine, la FMH et la Caisse des médecins. Le but de Newlndex est la création d'un pool de données pour l'ensemble de la Suisse, afin de générer des données propres aux médecins pour les négociations tarifaires avec les assureurs et de fournir aux médecins un instrument pour d'éventuelles procédures d'économicité.

Parmi les projets réussis de NewIndex AG, on trouve le calcul des valeurs de point tarifaire initiales et la mise en place des TrustCenters.
}

Ces dernières années, les exigences vis-à-vis du corps médical suisse se sont nettement accrues et l'évolution des tarifs réclame toujours plus d'économies alors que la demande augmente. Ernst Gähler, vice-président de la FMH, est persuadé que le corps médical ne peut réaliser ces économies que grâce à ses propres données. «Le pool de données détaillé de NewIndex offre au corps médical suisse une occasion unique d'occuper une place de leader», souligne le Dr Renato Laffranchi, directeur général de NewIndex AG, "car aucune autre institution ne possède de relevés de données aussi détaillés.»

\section{OBELISC (OBjective Evaluation and Leadership In Scientific Health Data Collection)} Afin de pouvoir affronter les défis croissants, et aussi remplir les tâches supplémentaires créées dans ce contexte, NewIndex a besoin de l'aide du corps médical suisse pour créer les compétences et les bases de données nécessaires indispensables à l'exploitation des données. La création du collectif spécial OBELISC permet à NewIndex de pratiquer des analyses et des interprétations à partir de données désagrégées ou non condensées. En considération d'intérêts de protection des données, toutes les publications basées sur ces données contiendront seulement des données agrégées, ne permettant absolument pas d'identifier le médecin ou le patient. Il s'agit donc de créer un nouveau lien contractuel entre le médecin et NewIndex, garantissant la sécurité au médecin: tout médecin participant autorise NewIndex à utiliser ses données désagrégées et NewIndex s'engage à une utilisation transparente. Le contrat existant entre le médecin et le TrustCenter, ainsi que les anciens contrôles et procédures (organe NAKO) restent inchangés et demeurent valides. Aucun coût supplémentaire n'est généré pour les médecins participants.

Les personnes présentes à la conférence du 29.3.2012 sont unanimes: les interprétations de 


\section{Utilisation des données} Hier, aujourd'hui et demain

Introduction TARMED et phase NC

$>$ Défense de la valeur de point tarifaire

initiale et de la parité des données

CPP

$>$ Pilotage de la VPT cantonale et

facteurs de prestations

TARVISION

$>$ Révision de la structure tarifaire TARMED

nDMA

$>$ Modèle innovateur de dispensation des médicaments par le médecin indépendant de la marge
Centre de compétences

$>$ Tarifs et contrats du corps médical

Réseaux et nouveaux modèles de soins

$>$ Contrats avec les assureurs et parité des données

Recherche en matière de soins

$>$ Avenir du système de santé suisse

\section{newi[ndex}

données déjà existantes doivent à l'avenir être mieux utilisées, afin d'améliorer les prestations dans le système de santé suisse et de défendre au mieux les intérêts du corps médical suisse. Car finalement, l'important, c'est le bénéfice pour le patient!

\section{Données désagrégées et leur importance dans le système de santé suisse}

Lors de la conférence, le collectif spécial OBELISC a reçu un accueil très positif. L'exposé du Dr Andreas Bänziger, collaborateur scientifique à la Haute école de sciences appliquées de Zurich (ZHAW), illustre à l'exemple des «Besoins régionaux de soins psychiatriques» l'importance des données non condensées, qui, contrairement aux données du pool de santésuisse, permettent une analyse détaillée du nombre de patients à l'échelle cantonale. Bänziger souligne l'importance d'un accès facile aux données, d'une liberté d'échange des données et d'une couverture complète des relevés.

Le Dr Peter Wiedersheim, coprésident de la Conférence des sociétés cantonales de médecine, voit un potentiel énorme dans les possibilités élargies d'utilisation des données de facturation collectées par le biais des TrustCenters des médecins: analyse détaillée des données par canton, contrôle de certaines prestations (par ex. FSME), observation des évolutions au sein des sociétés de discipline médicale ou du comportement de facturation dans les cantons. «Nous disposons déjà d'un excellent pool de données - nous devons absolument élargir cette base et l'utiliser de façon optimale, car à l'avenir l'importance des données propres ne cessera d'augmenter pour le corps médical», dit Wiedersheim, qui appelle les médecins à soutenir NewIndex et le projet OBELISC.

Le Dr Jürg Lareida, délégué tarifaire du canton d'Argovie, illustre l'importance des données des médecins pour le canton et l'argumentation politique, à partir de la situation dans le canton d'Argovie. Sur la base de différentes sources de données, une image très complète a pu être montrée pour le canton: les cabinets de premier recours sont confrontés à une hausse des coûts (des salaires en premier lieu) et une baisse des bénéfices, accompagnées d'une augmentation de la charge de travail. D’après Lareida, grâce au pool de données de NewIndex, les coûts par ex. peuvent être séparés selon l'âge des patients, permettant une vue différenciée. Selon Anton Prantl, directeur de la Caisse des médecins, «Compte-tenu des enjeux futurs se présentant au corps médical, il sera nécessaire de discuter de nouvelles solutions financières à long terme pour la collecte et l'utilisation des données.»

\section{Prise de position de la Dresse Franziska Zogga et du Dr Jürg Nadig}

a Membre du comité de Médecins de famille Suisse (Association des médecins de famille et de l'enfance Suisse) et vice-présidente de la Société de médecine du canton de Zoug

b président de la Swiss Federation of Specialities in Medicine (SFSM)
Pourquoi est-il important pour le corps médical suisse de disposer de ses propres données?

Zogg: Seules nos propres données permettent de contrôler la plausibilité des statistiques des autres, par exemple de santésuisse, et de réfuter les fausses affirmations. Les données propres aident aussi à identifier les tendances de façon précoce et à agir en conséquence.

Nadig: Grâce aux données de NewIndex, on a pu montrer avec l'exemple des oncologues médicaux que le modèle employé par santésuisse pour l'évaluation de l'économicité n'est pas en mesure de représenter les particularités des cabinets et de constituer un collectif homogène. Le modèle élaboré par la société de discipline a été validé avec succès par les données des médecins et permet, avec des positions marqueurs pertinentes, de constituer un collectif homogène.

\section{Quelle valeur a OBELISC pour les médecins} spécialistes et pour vous personnellement, et où voyez-vous le potentiel des collectes de données dans les défis futurs du système de santésuisse? Zogg: OBELISC est pour moi un saut quantique: il permet de faire des affirmations sur le contenu des consultations en relation avec certaines positions tarifaires ou avec la Reason for Encounter RFE. Ceci 
nous permet d'exploiter vraiment l'énorme potentiel de nos données. Par exemple: quelle position contient une visite dans quelle région du pays en moyenne? Qu'est-ce qui différencie les consultations en campagne des consultations en ville? Nous ne compterons pas seulement le nombre de consultations pour un code RFE donné, mais nous pourrons aussi évaluer les prestations fournies au cours de ces consultations.
Collectif spécial OBELISC: participez! En adhérant à OBELISC et en signant la déclaration correspondante de mise à disposition de vos données, vous permettez à Newlndex de réaliser d'importantes interprétations pour le compte et pour le bien du corps médical.

Si vous voulez vous aussi participer à ce projet d'avenir, la déclaration d'adhésion se trouve sous www.newindex.ch/f/obelisc.asp

\section{«Seules nos propres données permettent de contrôler la plausibilité des statistiques des autres, par exemple de santésuisse, et de réfuter les fausses affirmations.»}

Nadig: OBELISC permet une analyse approfondie des données et ainsi plus de transparence en ce qui concerne les particularités des cabinets. Les données désagrégées permettent aussi d'occuper le terrain de la recherche en matière de soins. Avec la pression croissante des coûts, il sera de plus en plus important pour le corps médical de disposer dans ce domaine aussi de ses propres données. Nous ne pouvons commencer à contrôler les processus que si nous connaissons la qualité et les coûts totaux d'une chaîne de soins.
Quels avantages voyez-vous dans OBELISC et pour quelles raisons pouvez-vous recommander ce projet à vos collègues?

Nadig: Après que ces données nous ont permis de mener le jeu dans les procédures d'économicité, nous devrions exiger un rôle de leader aussi dans la recherche en matière de soins et ne pas le laisser aux économistes. Nous, médecins et sociétés de discipline médicale, sommes les plus à même d'analyser les données. Si nous ne possédons pas nos propres données, les autres groupes interprèteront leurs données dans leur propre intérêt. 